\title{
On Poincaré-Friedrichs Type Inequalities for the Broken Sobolev Space $\mathrm{W}^{2,1}$
}

\author{
R. H. W. Hoppe $\mathrm{1}^{1,2, *}$ \\ ${ }^{1}$ Department of Mathematics, University of Houston, USA \\ ${ }^{2}$ Department of Mathematics, University of Augsburg, Germany
}

Received 17 April 2020; Accepted (in revised version) 5 August 2020

\begin{abstract}
We are concerned with the derivation of Poincaré-Friedrichs type inequalities in the broken Sobolev space $W^{2,1}\left(\Omega ; \mathcal{T}_{h}\right)$ with respect to a geometrically conforming, simplicial triagulation $\mathcal{T}_{h}$ of a bounded Lipschitz domain $\Omega$ in $\mathbb{R}^{d}, d \in \mathbb{N}$. Such inequalities are of interest in the numerical analysis of nonconforming finite element discretizations such as $\mathrm{C}^{0}$ Discontinuous Galerkin $\left(\mathrm{C}^{0} \mathrm{DG}\right)$ approximations of minimization problems in the Sobolev space $W^{2,1}(\Omega)$, or more generally, in the Banach space $B V^{2}(\Omega)$ of functions of bounded second order total variation. As an application, we consider a $C^{0} D G$ approximation of a minimization problem in $B V^{2}(\Omega)$ which is useful for texture analysis and management in image restoration.
\end{abstract}

AMS subject classifications: 65K10, 65N30, 68U10

Key words: Poincaré-Friedrichs inequalities, broken Sobolev spaces, $\mathrm{C}^{0}$ Discontinuous Galerkin approximation, image processing.

\section{Introduction}

Poincaré-Friedrichs type inequalities for broken Sobolev spaces play an important role in the numerical analysis of nonconforming finite element discretizations of minimization problems in Sobolev spaces and associated partial differential equations (cf., e.g., $[6-8,12,13])$. In this paper, given a bounded Lipschitz domain $\Omega$ in $\mathbb{R}^{d}, d \in \mathbb{N}$, we derive such inequalities for the broken Sobolev space $W^{2,1}\left(\Omega ; \mathcal{T}_{h}\right)$ with respect to a geometrically conforming, simplicial triangulation $\mathcal{T}_{h}$ of $\Omega$. They are based on PoincaréFriedrichs type inequalities for the space $B V^{2}(\Omega)$ of functions of bounded second order total variation $[3,4]$. As an application, we consider a $C^{0}$ Discontinuous Galerkin $\left(\mathrm{C}^{0} \mathrm{DG}\right)$ approximation of a minimization problem in $B V^{2}(\Omega)$ which is used in image processing for texture analysis and management.

\footnotetext{
${ }^{*}$ Corresponding author. Email addresses: rohop@math.uh.edu, rhhoppe@central.uh.edu (R. Hoppe) 
The paper is organized as follows: In Section 2, we introduce the Banach spaces $B V(\Omega)$ and $B V^{2}(\Omega)$ of functions of bounded first and second order total variation and recall some of its properties that pertain to the derivation of the Poincaré-Friedrichs type inequalities, whereas Section 3 is devoted to Poincaré-Friedrichs type inequalities in $B V^{2}(\Omega)$. In Section 4, we define the broken Sobolev space $W^{2,1}\left(\Omega ; \mathcal{T}_{h}\right)$ in terms of a broken Hessian involving a recovery operator from the broken Sobolev space $W^{2,1}\left(\Omega ; \mathcal{T}_{h}\right)$ into the linear space of $d \times d$ matrices with element-wise polynomial entries. We prove the boundedness of the recovery operator in the $\mathrm{L}^{1}$ norm (Theorem 4.1). In Section 5 , we first show that for functions in $W^{2,1}\left(\Omega ; \mathcal{T}_{h}\right)$ the second order total variation can be bounded from above by the $W^{2,1}\left(\Omega ; \mathcal{T}_{h}\right)$ seminorm and obtain a compactness result in the sense that bounded sequences in $W^{2,1}\left(\Omega ; \mathcal{T}_{h}\right)$ contain a subsequence converging weakly* in $B V^{2}(\Omega)$ (Theorem 5.1). We then derive two Poincaré-Friedrichs type inequalities for the broken Sobolev space $W^{2,1}\left(\Omega ; \mathcal{T}_{h}\right)$ (Theorem 5.2). Finally, in Section 6 we consider the $C^{0} D G$ approximation of a minimization problem in $B V^{2}(\Omega)$ which can be applied to texture analysis and management in image restoration $[4,5]$ and prove that the sequence of $\mathrm{C}^{0} \mathrm{DG}$ approximations in $W^{2,1}\left(\Omega ; \mathcal{T}_{h}\right)$ contains a subsequence converging weakly* in $B V^{2}(\Omega)$ to a solution of the original minimization problem (Theorem 6.1).

\section{Functions of bounded first and second order total variation}

For a bounded Lipschitz domain $\Omega \subset \mathbb{R}^{d}, d \in \mathbb{N}$, with boundary $\Gamma=\partial \Omega$ we refer to $C_{0}^{m}(\Omega), 0 \leq m \leq \infty$, as the Banach space of $m$-times continuously differentiable scalar functions with compact support in $\Omega$. Likewise, $C_{0}^{m}\left(\Omega ; \mathbb{R}^{d}\right), 0 \leq m \leq \infty$, stands for the Banach space of $m$-times continuously differentiable vector-valued functions with compact support in $\Omega$ and $C_{0}^{m}\left(\Omega ; \mathbb{R}^{d \times d}\right), 0 \leq m \leq \infty$, for the Banach space of $m$-times continuously differentiable matrix-valued functions with compact support in $\Omega$.

Moreover, we will use standard notation from Lebesgue and Sobolev space theory [14]. In particular, for Lipschitz subsets $D \subseteq \bar{\Omega}$ and $1 \leq p \leq \infty$ we denote the $L^{p}$-norm by $\|\left.\cdot\right|_{L^{p}(D)}$. We further refer to $W^{m, p}(D), m \in \mathbb{N}$, as the Sobolev spaces with norm $\|\cdot\|_{W^{m, p}(D)}$, and seminorm $|\cdot|_{W^{m, p}(D)}$, and to $W^{m-\frac{1}{p}, p}\left(\Gamma^{\prime}\right), \Gamma^{\prime} \subset \partial D$, as the associated trace spaces. $W_{0}^{m, p}(D)$ stands for the closure of $C_{0}^{\infty}(D)$ in the $W^{m, p}$-norm. Sobolev spaces $W^{s, p}(D)$ with broken index $s \in \mathbb{R}_{+}$are defined by interpolation. In case $p=2$ we will write $H^{m}(D)$ instead of $W^{m, 2}(D)$. The spaces $L^{2}(D)$ and $H^{m}(D)$ are Hilbert spaces with inner products denoted by $(\cdot, \cdot)_{L^{2}(D)}$ and $(\cdot, \cdot)_{H^{m}(D)}$. Further, $H^{-m}(D)$ refers to the dual space of $H_{0}^{m}(D)$ with $\langle\cdot, \cdot\rangle_{m, D}$ denoting the dual product.

The spaces $L^{p}\left(D ; \mathbb{R}^{d}\right), 1 \leq p \leq \infty$, stand for the Banach spaces of vector-valued functions $\underline{\mathbf{q}}=\left(q_{1}, \ldots, q_{d}\right)^{T}$ with norm $\|\underline{\mathbf{q}}\|_{L^{p}\left(D ; \mathbb{R}^{d}\right)}:=\left(\int_{D}|\underline{\mathbf{q}}|^{p} d x\right)^{\frac{1}{p}}$ for $1 \leq p<\infty$, where $|\underline{\mathbf{q}}|:=(\underline{\mathbf{q}} \cdot \underline{\mathbf{q}})^{\frac{1}{2}}$ and $\underline{\mathbf{p}} \cdot \underline{\mathbf{q}}=\sum_{i=1}^{d} p_{i} q_{i}$, and $\|\underline{\mathbf{q}}\|_{L^{\infty}\left(D ; \mathbb{R}^{d}\right)}:=\max _{1 \leq i \leq d}\left\|q_{i}\right\|_{L^{\infty}(D)}$ for $p=\infty$. Likewise, we refer to $L^{p}\left(\Omega ; \mathbb{R}^{d \times d}\right), 1 \leq p \leq \infty$, as the Banach spaces of matrix-valued functions $\underline{\underline{\mathbf{q}}}=\left(q_{i j}\right)_{i, j=1}^{d}$ with norm $\|\underline{\underline{\mathbf{q}}}\|_{L^{p}\left(D ; \mathbb{R}^{d \times d}\right)}:=\left(\int_{D}|\underline{\underline{\mathbf{q}}}|^{p} d x\right)^{\frac{1}{p}}$ for 
$1 \leq p<\infty$, where $|\underline{\underline{\mathbf{q}}}|:=(\underline{\underline{\mathbf{q}}}: \underline{\underline{\mathbf{q}}})^{\frac{1}{2}}$ and $\underline{\underline{\mathbf{p}}}: \underline{\underline{\mathbf{q}}}:=\sum_{i, j=1}^{d} p_{i j} q_{i j}$, and $\|\underline{\mathbf{q}}\|_{L^{\infty}\left(D ; \mathbb{R}^{d \times d}\right)}:=$ $\max _{1 \leq i, j \leq d}\left\|q_{i j}\right\|_{L^{\infty}(D)}$ for $p=\infty$.

Further, we denote by $\mathcal{M}\left(\Omega ; \mathbb{R}^{d}\right), d \in \mathbb{N}$, the Banach space of vector-valued bounded Radon measures $\boldsymbol{\mu}=\left(\mu_{1}, \ldots, \mu_{d}\right)$ equipped with the total variation norm

$$
|\boldsymbol{\mu}|(\Omega):=\sup \left\{\sum_{n=1}^{\infty}\left|\boldsymbol{\mu}\left(\Omega_{n}\right)\right| \mid \Omega=\bigcup_{n=1}^{\infty} \Omega_{n}, \Omega_{n} \cap \Omega_{m}=\emptyset \text { for } n \neq m\right\} .
$$

In view of the Riesz representation theorem $\mathcal{M}\left(\Omega ; \mathbb{R}^{d}\right)$ is the dual space of $C_{0}\left(\Omega ; \mathbb{R}^{d}\right)$ with the duality pairing

$$
\langle\boldsymbol{\mu}, \mathbf{q}\rangle_{\mathcal{M}, C_{0}}:=\int_{\Omega} \mathbf{q} d \boldsymbol{\mu}=\sum_{i=1}^{d} \int_{\Omega} q_{i} d \mu_{i} .
$$

A sequence $\left\{\boldsymbol{\mu}_{n}\right\}_{\mathbb{N}}$ of Radon measures $\boldsymbol{\mu}_{n} \in \mathcal{M}\left(\Omega ; \mathbb{R}^{d}\right), n \in \mathbb{N}$, is said to converge weakly* to $\boldsymbol{\mu} \in \mathcal{M}\left(\Omega ; \mathbb{R}^{d}\right)\left(\boldsymbol{\mu}_{n} \rightarrow^{*} \boldsymbol{\mu}(n \rightarrow \infty)\right)$ if

$$
\left\langle\boldsymbol{\mu}_{n}, \mathbf{q}\right\rangle_{\mathcal{M}, C_{0}} \rightarrow\langle\boldsymbol{\mu}, \mathbf{q}\rangle_{\mathcal{M}, C_{0}}(n \rightarrow \infty) \text { for all } \mathbf{q} \in C_{0}^{\infty}\left(\Omega ; \mathbb{R}^{d}\right) .
$$

Likewise, we refer to $\mathcal{M}\left(\Omega ; \mathbb{R}^{d \times d}\right), d \in \mathbb{N}$, as the Banach space of matrix-valued bounded Radon measures $\underline{\underline{\mu}}=\left(\mu_{i j}\right)_{i, j=1}^{d}$ equipped with the total variation norm

$$
\underline{\underline{\underline{\mu}}} \mid(\Omega):=\sup \left\{\sum_{n=1}^{\infty} \underline{\underline{\mu}}\left(\Omega_{n}\right)|| \Omega=\bigcup_{n=1}^{\infty} \Omega_{n}, \Omega_{n} \cap \Omega_{m}=\emptyset \text { for } n \neq m\right\} \text {. }
$$

The space $\mathcal{M}\left(\Omega ; \mathbb{R}^{d \times d}\right)$ is the dual space of $C_{0}\left(\Omega ; \mathbb{R}^{d \times d}\right)$ with the duality pairing

$$
\langle\underline{\underline{\mu}}, \underline{\underline{\mathbf{q}}}\rangle_{\mathcal{M}, C_{0}}:=\int_{\Omega} \underline{\underline{\mathbf{q}}} d \underline{\underline{\mu}}=\sum_{i, j=1}^{d} \int_{\Omega} q_{i j} d \mu_{i j}
$$

A sequence $\left\{\underline{\underline{\mu}}_{n}\right\}_{\mathbb{N}}$ of Radon measures $\underline{\underline{\mu}}_{n} \in \mathcal{M}\left(\Omega ; \mathbb{R}^{d \times d}\right), n \in \mathbb{N}$, is said to converge

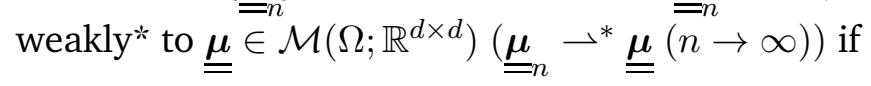

$$
\left\langle\underline{\underline{\mu}}_{n}, \underline{\underline{\mathbf{q}}}\right\rangle_{\mathcal{M}, C_{0}} \rightarrow\langle\underline{\underline{\mu}}, \underline{\underline{\mathbf{q}}}\rangle_{\mathcal{M}, C_{0}}(n \rightarrow \infty) \quad \text { for all } \quad \underline{\underline{\mathbf{q}}} \in C_{0}^{\infty}\left(\Omega ; \mathbb{R}^{d \times d}\right)
$$

A function $u \in L^{1}(\Omega)$ is said to be of (first order) bounded total variation if its distributional derivative $D u$ satisfies $D u \in \mathcal{M}\left(\Omega ; \mathbb{R}^{d}\right)$, i.e., for all $\underline{\varphi} \in C_{0}^{1}\left(\Omega ; \mathbb{R}^{d}\right)$ we have

$$
-\int_{\Omega} u \nabla \cdot \underline{\varphi} d x=\int_{\Omega} \underline{\varphi} \cdot d D u<\infty .
$$


The (first order) total variation of $u$ is defined as follows

$$
|D u|(\Omega):=\sup \left\{-\int_{\Omega} u \nabla \cdot \underline{\varphi} d x\left|\underline{\varphi} \in C_{0}^{1}\left(\Omega ; \mathbb{R}^{d}\right),\right| \underline{\varphi}(x) \mid \leq 1, x \in \Omega\right\} .
$$

We denote by $B V(\Omega)$ the Banach space of functions $u \in L^{1}(\Omega)$ such that $|D u|(\Omega)<\infty$ equipped with the norm

$$
\|u\|_{B V(\Omega)}:=\|u\|_{L^{1}(\Omega)}+|D u|(\Omega) .
$$

For vector-valued functions $\underline{\mathbf{u}}=\left(u_{1}, \ldots, u_{d}\right)^{T} \in L^{1}\left(\Omega ; \mathbb{R}^{d}\right)$ we refer to $B V\left(\Omega ; \mathbb{R}^{d}\right)$ as the Banach space of functions with $u_{i} \in B V(\Omega), 1 \leq i \leq d$.

Clearly, we have $W^{1,1}(\Omega) \subset B V(\Omega)$ and $u \in W^{1,1}(\Omega)$ iff $u \in L^{1}(\Omega)$ and $D u$ is absolutely continuous with respect to the Lebesgue measure. In particular, we have the Lebesgue-Radon-Nikodym decomposition

$$
D u=\nabla u+D_{s} u,
$$

where $\nabla u \in L^{1}\left(\Omega ; \mathbb{R}^{d}\right)$ is called the approximate gradient of $u$ and $D_{s} u \in \mathcal{M}\left(\Omega ; \mathbb{R}^{d}\right)$ is said to be the singular part of the derivative.

A sequence $\left\{u_{n}\right\}_{\mathbb{N}}$ of functions $u_{n} \in B V(\Omega), n \in \mathbb{N}$, is said to converge weakly* to $u \in B V(\Omega)$ if $u_{n} \rightarrow u$ in $L^{1}(\Omega)$ and $D u_{n} \rightarrow^{*} D u$ in $\mathcal{M}\left(\Omega ; \mathbb{R}^{d}\right)$ as $\mathbb{N} \ni n \rightarrow \infty$.

The following results on $B V(\Omega)$ are listed without proofs which can be found in [1] and [2].

Lemma 2.1. Let $\left\{u_{n}\right\}_{\mathbb{N}}$ be a uniformly bounded sequence of functions $u_{n} \in B V(\Omega), n \in$ $\mathbb{N}$, i.e.,

$$
\left\|u_{n}\right\|_{B V(\Omega)} \leq C, \quad n \in \mathbb{N}
$$

for some $C>0$. Then there exist a subsequence $\mathbb{N}^{\prime} \subset \mathbb{N}$ and $u \in B V(\Omega)$ such that

$$
u_{n} \rightarrow^{*} u \text { in } B V(\Omega) \text { as } \quad \mathbb{N}^{\prime} \ni n \rightarrow \infty .
$$

We have the following embedding and lower semicontinuity results.

Theorem 2.1. (i) The space $B V(\Omega)$ is continuously embedded in $L^{p}(\Omega)$ for $p \leq \frac{d}{d-1}$. It is compactly embedded in $L^{p}(\Omega)$ for $p<\frac{d}{d-1}$.

(ii) The mapping $u \longmapsto|D(u)|(\Omega)$ is lower semicontinuous from $B V(\Omega)$ into $\mathbb{R}_{+}$, i.e., if $\left\{u_{n}\right\}_{\mathbb{N}}$ is a sequence of functions $u_{n} \in B V(\Omega), n \in \mathbb{N}$, and $u \in B V(\Omega)$ such that $u_{n} \rightarrow^{*} u(\mathbb{N} \ni n \rightarrow \infty)$ in $B V(\Omega)$, then it holds

$$
|D(u)|(\Omega) \leq \lim \inf _{n \rightarrow \infty}\left|D\left(u_{n}\right)\right|(\Omega) .
$$

A sequence $\left\{u_{n}\right\}_{\mathbb{N}}$ of functions $u_{n} \in B V(\Omega), n \in \mathbb{N}$, is said to converge strictly to $u \in B V(\Omega)$ if $u_{n} \rightarrow u$ in $L^{1}(\Omega)$ and $\left|D u_{n}\right|(\Omega) \rightarrow|D u|(\Omega)$ as $\mathbb{N} \ni n \rightarrow \infty$. 
Lemma 2.2. The space $C^{\infty}(\bar{\Omega})$ is dense in $B V(\Omega)$ in the sense that for every $u \in B V(\Omega)$ there exists a sequence $\left\{u_{n}\right\}_{\mathbb{N}}$ of functions $u_{n} \in C^{\infty}(\bar{\Omega})$ such that $\left\{u_{n}\right\}_{\mathbb{N}}$ converges strictly to $u$.

Functions $u \in B V(\Omega)$ have a trace $\left.u\right|_{\Gamma} \in L^{1}(\Gamma)$. The trace mapping $T: B V(\Omega) \rightarrow$ $L^{1}(\Gamma)$ is linear, continuous from $B V(\Omega)$ endowed with the strict topology to $L^{1}(\Gamma)$ equipped with the strong topology (cf., e.g., [2, Theorem 10.2.2]). The subspace $B V_{0}(\Omega)$ of $B V(\Omega)$ is the kernel of the trace mapping $T$. It is a Banach space equipped with the induced norm.

A function $u \in W^{1,1}(\Omega)$ is said to be of bounded second order total variation if its distributional derivative $D^{2} u$ satisfies $D^{2} u \in \mathcal{M}\left(\Omega ; \mathbb{R}^{d \times d}\right)$, i.e., for all $\underline{\underline{\varphi}} \in C_{0}^{1}\left(\Omega ; \mathbb{R}^{d \times d}\right)$ we have

$$
-\int_{\Omega} \nabla u \cdot \nabla \cdot \underline{\underline{\varphi}} d x=\int_{\Omega} \underline{\underline{\varphi}} \cdot d D^{2} u<\infty
$$

The second order total variation of $u$ is defined as follows

$$
\left|D^{2} u\right|(\Omega):=\sup \left\{-\int_{\Omega} \nabla u \cdot \nabla \cdot \underline{\underline{\varphi}} d x\left|\underline{\underline{\varphi}} \in C_{0}^{2}\left(\Omega ; \mathbb{R}^{d \times d}\right),\right| \underline{\underline{\varphi}}(x) \mid \leq 1, x \in \Omega\right\} .
$$

We denote by $B V^{2}(\Omega)$ the Banach space of functions of bounded second order variation equipped with the norm (cf., e.g., [10])

$$
\|u\|_{B V^{2}(\Omega)}=\|u\|_{W^{1,1}(\Omega)}+\left|D^{2} u\right|(\Omega) .
$$

A function $u$ belongs to $B V^{2}(\Omega)$ iff $u \in W^{1,1}(\Omega)$ and $\nabla u \in B V\left(\Omega ; \mathbb{R}^{d}\right)$, i.e., $\frac{\partial u}{\partial x_{i}} \in$ $B V(\Omega), 1 \leq i \leq d$. Moreover, for $u \in B V^{2}(\Omega)$ it holds

$$
\left|D^{2} u\right|(\Omega) \leq \sum_{i=1}^{d}\left|\frac{\partial u}{\partial x_{i}}\right|(\Omega) \leq d\left|D^{2} u\right|(\Omega) .
$$

Obviously, we have $W^{2,1}(\Omega) \subset B V^{2}(\Omega)$ and $u \in W^{2,1}(\Omega)$ iff $u \in W^{1,1}(\Omega)$ and $D^{2} u$ is absolutely continuous with respect to the Lebesgue measure. The following LebesgueRadon-Nikodym type decomposition holds true

$$
D^{2} u=\nabla^{2} u+D_{s}^{2} u
$$

where $\nabla^{2} u \in L^{1}\left(\Omega ; \mathbb{R}^{d \times d}\right)$ is referred to as the approximate Hessian of $u$ and $D_{s}^{2} u \in$ $\mathcal{M}\left(\Omega ; \mathbb{R}^{d \times d}\right)$ is called the singular part of the Hessian.

A sequence $\left\{u_{n}\right\}_{\mathbb{N}}$ of functions $u_{n} \in B V^{2}(\Omega), n \in \mathbb{N}$, is said to converge weakly* to $u \in B V^{2}(\Omega)$ if $u_{n} \rightarrow u$ in $W^{1,1}(\Omega)$ and $D^{2} u_{n} \rightarrow^{*} D^{2} u$ in $\mathcal{M}\left(\Omega ; \mathbb{R}^{d \times d}\right)$ as $\mathbb{N} \ni n \rightarrow \infty$.

The following results on $B V^{2}(\Omega)$ are listed without proofs which can be found in $[10]$. 
Theorem 2.2. Let $\left\{u_{n}\right\}_{\mathbb{N}}$ be a uniformly bounded sequence of functions $u_{n} \in B V^{2}(\Omega), n \in$ $\mathbb{N}$, i.e.,

$$
\left\|u_{n}\right\|_{B V^{2}(\Omega)} \leq C, \quad n \in \mathbb{N}
$$

for some $C>0$. Then there exist a subsequence $\mathbb{N}^{\prime} \subset \mathbb{N}$ and $u \in B V^{2}(\Omega)$ such that

$$
u_{n} \rightarrow^{*} u \text { in } B V^{2}(\Omega) \text { as } \quad \mathbb{N}^{\prime} \ni n \rightarrow \infty .
$$

We have the following embedding and lower semicontinuity results.

Theorem 2.3. (i) The space $B V^{2}(\Omega)$ is continuously embedded in $W^{1, p}(\Omega)$ for $p \leq \frac{d}{d-1}$. It is compactly embedded in $W^{1, p}(\Omega)$ for $p<\frac{d}{d-1}$.

(ii) The mapping $u \longmapsto\left|D^{2}(u)\right|(\Omega)$ is lower semicontinuous from $B V^{2}(\Omega)$ into $\mathbb{R}_{+}$, i.e., if $\left\{u_{n}\right\}_{\mathbb{N}}$ is a sequence of functions $u_{n} \in B V^{2}(\Omega), n \in \mathbb{N}$, and $u \in B V^{2}(\Omega)$ such that $u_{n} \rightarrow^{*} u(\mathbb{N} \ni n \rightarrow \infty)$ in $B V^{2}(\Omega)$, then it holds

$$
\left|D^{2}(u)\right|(\Omega) \leq \lim \inf _{n \rightarrow \infty}\left|D^{2}\left(u_{n}\right)\right|(\Omega) .
$$

A sequence $\left\{u_{n}\right\}_{\mathbb{N}}$ of functions $u_{n} \in B V^{2}(\Omega), n \in \mathbb{N}$, is said to converge strictly to $u \in B V^{2}(\Omega)$ if $u_{n} \rightarrow u$ in $W^{1,1}(\Omega)$ and $\left|D^{2} u_{n}\right|(\Omega) \rightarrow\left|D^{2} u\right|(\Omega)$ as $\mathbb{N} \ni n \rightarrow \infty$.

Lemma 2.3. The space $C^{\infty}(\bar{\Omega})$ is dense in $B V^{2}(\Omega)$ in the sense that for every $u \in B V^{2}(\Omega)$ there exists a sequence $\left\{u_{n}\right\}_{\mathbb{N}}$ of functions $u_{n} \in C^{\infty}(\bar{\Omega})$ such that $\left\{u_{n}\right\}_{\mathbb{N}}$ converges strictly to $u$.

\section{Poincaré-Friedrichs type inequalities in $B V^{2}(\Omega)$}

Since $B V^{2}(\Omega) \subset B V(\Omega)$, the trace mapping $T: B V^{2}(\Omega) \rightarrow L^{1}(\Gamma)$ is well defined. We set $B V_{0}^{2}(\Omega):=\left\{v \in B V^{2}(\Omega)|v|_{\Gamma}=0\right\}$ and further define

$$
B V_{m}^{2}(\Omega):=\left\{v \in B V^{2}(\Omega) \mid \int_{\Omega} \frac{\partial v}{\partial x_{i}} d x=0,1 \leq i \leq d\right\} .
$$

Lemma 3.1. The trace mapping $T: B V^{2}(\Omega) \rightarrow L^{1}(\Gamma)$ is compact.

Proof. In view of Theorem 2.3 the Banach space $B V^{2}(\Omega)$ is compactly embedded in $W^{1,1}(\Omega)$. On the other hand, the trace mapping $T: W^{1,1}(\Omega) \rightarrow L^{1}(\Gamma)$ is continuous.

We briefly review the following Poincaré-Friedrichs type inequalities in $B V^{2}(\Omega)$ from [4].

Lemma 3.2. There exist generic constants $C_{i}>0,1 \leq i \leq 2$, such that

$$
\begin{array}{ll}
|u|_{W^{1,1}(\Omega)} \leq C_{1}\left|D^{2}(u)\right|(\Omega), & u \in B V_{0}^{2}(\Omega), \\
|u|_{W^{1,1}(\Omega)} \leq C_{2}\left|D^{2}(u)\right|(\Omega), & u \in B V_{m}^{2}(\Omega) .
\end{array}
$$


Proof. In [4, Lemma 3.1] it has been shown that

$$
\begin{array}{ll}
|D(u)|(\Omega) \leq C_{1}\left|D^{2}(u)\right|(\Omega), & u \in B V_{0}^{2}(\Omega), \\
|D(u)|(\Omega) \leq C_{2}\left|D^{2}(u)\right|(\Omega), & u \in B V_{m}^{2}(\Omega) .
\end{array}
$$

Since $\left.|D(u)|(\Omega)|=| u\right|_{W^{1,1}(\Omega)}$ for $u \in B V^{2}(\Omega)$, the assertion follows from (3.3).

\section{The broken Sobolev space $\mathrm{W}^{2,1}\left(\Omega ; \mathcal{T}_{\mathrm{h}}\right)$}

Let $\mathcal{T}_{h}$ be a geometrically conforming, simplicial triangulation of $\Omega$. We denote by $\mathcal{E}_{h}(\Omega)$ and $\mathcal{E}_{h}(\Gamma)$ the set of edges of $\mathcal{T}_{h}$ in the interior of $\Omega$ and on the boundary $\Gamma$, respectively, and set $\mathcal{E}_{h}:=\mathcal{E}_{h}(\Omega) \cup \mathcal{E}_{h}(\Gamma)$. For $K \in \mathcal{T}_{h}$ and $E \in \mathcal{E}_{h}$ we denote by $h_{K}$ and $h_{E}$ the diameter of $K$ and the length of $E$, and we set $h:=\max \left(h_{K} \mid K \in \mathcal{T}_{h}\right)$. We assume that the triangulation $\mathcal{T}_{h}$ is such there exist constants $0<c_{R} \leq C_{R}$ and $0<c_{S} \leq C_{S}$ such that for all $K \in \mathcal{T}_{h}$ it holds

$$
\begin{aligned}
& c_{R} h_{K} \leq h_{E} \leq C_{R} h_{K}, \quad E \in \mathcal{E}_{h}(\partial K), \\
& c_{S} h_{K}^{2} \leq \operatorname{meas}(K) \leq C_{S} h_{K}^{2} .
\end{aligned}
$$

For two quantities $A$ and $B$ we write $A \lesssim B$, if there exists a constant $C>0$ independent of $h$ such that $A \leq C B$. We further write $A \approx B$ iff $A \lesssim B$ and $B \lesssim A$.

We refer to $P_{m}(K), m \in \mathbb{N}$, the linear space of polynomials of degree $\leq m$ on $K$. We introduce $\underline{\underline{\mathbf{M}}}_{h}$ as the subspace of $L^{1}(\Omega)^{d \times d}$ such that the restriction of $\underline{\underline{\mathbf{q}}} \in L^{1}(\Omega)^{d \times d}$ to an element $K \in \mathcal{T}_{h}$ belongs to $P_{\ell}(K)^{d \times d}$ for some $\ell \in \mathbb{N}$, i.e., we have

$$
\underline{\underline{\mathbf{M}}}_{h}:=\left\{\underline{\underline{\mathbf{q}}} \in L^{1}(\Omega)^{d \times d}|\underline{\underline{\mathbf{q}}}|_{K} \in P_{\ell}(K)^{d \times d}, K \in \mathcal{T}_{h}\right\} \text {. }
$$

For interior edges $E \in \mathcal{E}_{h}(\Omega)$ such that $E=K_{+} \cap K_{-}, K_{ \pm} \in \mathcal{T}_{h}$ and boundary edges on $\Gamma$ we introduce the average and jump of $\underline{\underline{\mathbf{q}}} \in \underline{\underline{\mathbf{M}}}_{h}$ according to

$$
\begin{array}{r}
\{\underline{\underline{\mathbf{q}}}\}_{E}:= \begin{cases}\frac{1}{2}\left(\left.\underline{\underline{\mathbf{q}}}\right|_{E \cap K_{+}}+\left.\underline{\underline{\mathbf{q}}}\right|_{E \cap K_{-}}\right), & E \in \mathcal{E}_{h}(\Omega), \\
\left.\underline{\underline{\mathbf{q}}}\right|_{E}, & E \in \mathcal{E}_{h}(\Gamma),\end{cases} \\
\underline{\underline{\underline{\mathbf{q}}}]_{E}}:= \begin{cases}\left.\underline{\underline{\mathbf{q}}}\right|_{E \cap K_{+}}-\left.\underline{\underline{\mathbf{q}}}\right|_{E \cap K_{-}}, & E \in \mathcal{E}_{h}(\Omega), \\
\left.\underline{\underline{\mathbf{q}}}\right|_{E}, & E \in \mathcal{E}_{h}(\Gamma) .\end{cases}
\end{array}
$$

We further denote by $\mathbf{n}_{E}$ the unit normal vector on $E$ pointing in the direction from $K_{+}$to $K_{-}$.

For $k \in \mathbb{N}$ we define the broken Sobolev space $W^{2,1}\left(\Omega ; \mathcal{T}_{h}\right)$ according to

$$
W^{2,1}\left(\Omega ; \mathcal{T}_{h}\right):=\left\{u \in W^{1,1}(\Omega)|u|_{K} \in P_{k}(K), K \in \mathcal{T}_{h}\right\}
$$


equipped with the broken Sobolev seminorm

$$
|u|_{W^{2,1}\left(\Omega ; \mathcal{T}_{h}\right)}:=\sum_{K \in \mathcal{T}_{h}}\left\|\nabla^{2} u\right\|_{L^{1}\left(K ; \mathbb{R}^{d \times d}\right)}+\sum_{E \in \mathcal{E}_{h}} \int_{E}\left|\left[\nabla u \otimes \mathbf{n}_{E}\right]_{E}\right| d s,
$$

and the broken Sobolev norm

$$
\|u\|_{W^{2,1}\left(\Omega ; \mathcal{T}_{h}\right)}:=\|u\|_{W^{1,1}(\Omega)}+|u|_{W^{2,1}\left(\Omega ; \mathcal{T}_{h}\right)},
$$

where $\nabla u \otimes \mathbf{n}_{E}$ stands for the tensor product of $\nabla u$ and $\mathbf{n}_{E}$. For $u \in W^{2,1}\left(\Omega ; \mathcal{T}_{h}\right)$ we define the broken Hessian $\nabla_{h}^{2} u \in \underline{\underline{\mathbf{M}}}_{h}$ by means of

$$
\left.\nabla_{h}^{2} u\right|_{K}:=\left.\nabla^{2} u\right|_{K}, \quad K \in \mathcal{T}_{h} .
$$

Applying Green's formula, the distributional Hessian $D^{2} u$ of a function $u \in W^{2,1}\left(\Omega ; \mathcal{T}_{h}\right)$ satisfies

$$
<D^{2} u, \underline{\underline{\varphi}}>_{\mathcal{M}, C_{0}}=\sum_{K \in \mathcal{T}_{h}} \int_{K} \nabla^{2} u: \underline{\underline{\varphi}} d x-\sum_{E \in \mathcal{E}_{h}(\Omega)} \int_{E}\left[\nabla u \otimes \mathbf{n}_{E}\right]_{E}: \underline{\underline{\varphi}} d s,
$$

where $\underline{\underline{\varphi}} \in C_{0}^{\infty}\left(\Omega ; \mathbb{R}^{d \times d}\right)$. We construct a bulk representation of the jump contribution in (4.6) by defining the recovery operator $R: W^{2,1}\left(\Omega ; \mathcal{T}_{h}\right) \rightarrow \underline{\underline{\mathbf{M}}}_{h}$ according to

$$
\int_{\Omega} R(u): \underline{\underline{\varphi}} d x=-\sum_{E \in \mathcal{E}_{h}(\Omega)} \int_{E}\left[\nabla u \otimes \mathbf{n}_{E}\right]_{E}:\{\underline{\underline{\varphi}}\}_{E} d s, \quad \underline{\underline{\varphi}} \in \underline{\underline{M}}_{h},
$$

and set

$$
\nabla_{h, D G}^{2} u:=\nabla_{h}^{2} u+R(u) .
$$

The following auxiliary results will enable us to estimate the $L^{1}$ norm of $R(u)$. They are special cases of Lemma A1 and Lemma A2 from [8]. For completeness we will provide the proofs.

Lemma 4.1. Let the triangulation $\mathcal{T}_{h}$ satisfy the assumptions (4.1). Then there exists a constant $C>0$, independent of $h$, such that for $\underline{\underline{\varphi}} \in \underline{\underline{\mathbf{M}}}_{h}$ and any $K \in \mathcal{T}_{h}$ it holds

$$
\begin{gathered}
\|\underline{\underline{\varphi}}\|_{L^{\infty}\left(K ; \mathbb{R}^{d \times d}\right)} \leq C h_{K}^{-\frac{d}{2}}\|\underline{\underline{\varphi}}\|_{L^{2}\left(K ; \mathbb{R}^{d \times d}\right)}, \\
\|\underline{\underline{\varphi}}\|_{L^{1}\left(K ; \mathbb{R}^{d \times d}\right)} \leq C h_{K}^{\frac{d}{2}}\|\underline{\underline{\varphi}}\|_{L^{2}\left(K ; \mathbb{R}^{d \times d}\right)} .
\end{gathered}
$$

Proof. Let $K$ be an element of $\mathcal{T}_{h}, \hat{K}$ its reference element, and $B_{K}: \hat{K} \rightarrow K$ the associated d-linear mapping with $J_{K}:=\left|\operatorname{det} B_{K}\right|$ satisfying $J_{K} \approx h_{K}^{d}$. For $\underline{\underline{\varphi}} \in \underline{\underline{\mathbf{M}}}_{h}$ it holds

$$
\begin{aligned}
\|\underline{\underline{\varphi}}\|_{L^{2}\left(K ; \mathbb{R}^{d \times d}\right)} & =\int_{K}|\underline{\underline{\varphi}}(x)|^{2} d x=\int_{\hat{K}} J_{K}\left|\left(\underline{\underline{\varphi}} \circ B_{K}\right)(\hat{x})\right| d \hat{x} \\
& \approx h_{K}^{d} \int_{\hat{K}}\left|\left(\underline{\underline{\varphi}} \circ B_{K}\right)(\hat{x})\right| d \hat{x} .
\end{aligned}
$$


Poincaré-Friedrichs Inequalities for the Broken Sobolev Space $W^{2,1}$

Further, in view of the norm equivalence in finite dimensional spaces we have

$$
\left(\int_{\hat{K}}\left|\left(\underline{\underline{\varphi}} \circ B_{K}\right)(\hat{x})\right| d \hat{x}\right)^{\frac{1}{2}} \approx \sup _{\hat{x} \in \hat{K}}\left|\left(\underline{\underline{\varphi}} \circ B_{K}\right)(\hat{x})\right| \approx \sup _{x \in K}|\underline{\underline{\varphi}}(x)| .
$$

The assertion (4.9a) follows from (4.10) and (4.11). The proof of (4.9b) can be done similarly.

The preceding result allows us to deduce the following inf-sup property.

Lemma 4.2. Let the triangulation $\mathcal{T}_{h}$ satisfy the assumptions (4.1). Then there exists a constant $C>0$, independent of $h$, such that

$$
\inf _{u \in W^{2,1}\left(\Omega ; \mathcal{T}_{h}\right)} \sup _{\underline{\underline{\varphi}} \underline{\underline{\mathbf{M}}}_{h}}\left\{\left(\|R(u)\|_{L^{1}\left(\Omega ; \mathbb{R}^{d \times d}\right)}\|\underline{\underline{\varphi}}\|_{L^{\infty}\left(\Omega ; \mathbb{R}^{d \times d}\right)}\right)^{-1} \int_{\Omega} R(u): \underline{\underline{\varphi}} d x\right\} \geq C .
$$

Proof. For $u \in W^{2,1}\left(\Omega ; \mathcal{T}_{h}\right)$ we set $\underline{\underline{\mathbf{q}}}=\Pi_{\ell}\left(|R(u)|^{-1} R(u)\right)$, where $\Pi_{\ell}$ is the $\mathrm{L}^{2}$ projection onto $\underline{\underline{\mathbf{M}}}_{h}$ satisfying

$$
\int_{\Omega} R(u): \Pi_{\ell} \underline{\underline{\mathbf{q}}} d x=\int_{\Omega} R(u): \underline{\underline{\mathbf{q}}} d x=\|R(u)\|_{L^{1}\left(\Omega ; \mathbb{R}^{d \times d}\right)} .
$$

Hence, from (4.13) we deduce

$$
\begin{aligned}
& \inf _{u \in W^{2,1}\left(\Omega ; \mathcal{T}_{h}\right)} \sup _{\underline{\underline{\varphi}} \in \underline{\underline{\underline{M}}}_{h}\left\{\left(\|R(u)\|_{L^{1}\left(\Omega ; \mathbb{R}^{d \times d}\right)}\|\underline{\underline{\varphi}}\|_{L^{\infty}\left(\Omega ; \mathbb{R}^{d \times d}\right)}\right)^{-1} \int_{\Omega} R(u): \underline{\underline{\varphi}} d x\right\}} \\
\geq & \inf _{u \in W^{2,1}\left(\Omega ; \mathcal{T}_{h}\right)}\left\{\left(\|R(u)\|_{L^{1}\left(\Omega ; \mathbb{R}^{d \times d}\right)}\|\underline{\underline{\mathbf{q}}}\|_{L^{\infty}\left(\Omega ; \mathbb{R}^{d \times d}\right)}\right)^{-1} \int_{\Omega} R(u): \underline{\underline{\mathbf{q}}} d x\right\} \\
= & \inf _{u \in W^{2,1}\left(\Omega ; \mathcal{T}_{h}\right)}\left\|\Pi_{\ell}\left(|R(u)|^{-1} R(u)\right)\right\|_{L^{\infty}\left(\Omega ; \mathbb{R}^{d \times d}\right)}^{-1} .
\end{aligned}
$$

Moreover, applying (4.9b), for every $K \in \mathcal{T}_{h}$ we have

$$
\begin{aligned}
& \left\|\Pi_{\ell}\left(|R(u)|^{-1} R(u)\right)\right\|_{L^{2}\left(K ; \mathbb{R}^{d \times d}\right)}^{2} \\
= & \int_{K}|R(u)|^{-1} R(u) \Pi_{\ell}\left(|R(u)|^{-1} R(u)\right) d x \\
\leq & \left\||R(u)|^{-1} R(u)\right\|_{L^{\infty}\left(K ; \mathbb{R}^{d \times d}\right)}\left\|\Pi_{\ell}\left(|R(u)|^{-1} R(u)\right)\right\|_{L^{1}\left(K ; \mathbb{R}^{d \times d}\right)} \\
\leq & C h_{K}^{\frac{d}{2}}\left\|\Pi_{\ell}\left(|R(u)|^{-1} R(u)\right)\right\|_{L^{2}\left(K ; \mathbb{R}^{d \times d}\right)},
\end{aligned}
$$

whence

$$
\left\|\Pi_{\ell}\left(|R(u)|^{-1} R(u)\right)\right\|_{L^{2}\left(K ; \mathbb{R}^{d \times d}\right)} \leq C h_{K}^{\frac{d}{2}} .
$$


Now, (4.9a) and (4.15) imply

$$
\begin{aligned}
&\left\|\Pi_{\ell}\left(|R(u)|^{-1} R(u)\right)\right\|_{L^{\infty}\left(K ; \mathbb{R}^{d \times d}\right)} \\
& \leq C h_{K}^{-\frac{d}{2}}\left\|\Pi_{\ell}\left(|R(u)|^{-1} R(u)\right)\right\|_{L^{2}\left(K ; \mathbb{R}^{d \times d}\right)} \leq C,
\end{aligned}
$$

which readily gives

$$
\begin{aligned}
& \left\|\Pi_{\ell}\left(|R(u)|^{-1} R(u)\right)\right\|_{L^{\infty}\left(\Omega ; \mathbb{R}^{d \times d}\right)} \\
= & \sup _{K \in \mathcal{T}_{h}}\left\|\Pi_{\ell}\left(|R(u)|^{-1} R(u)\right)\right\|_{L^{\infty}\left(K ; \mathbb{R}^{d \times d}\right)} \leq C .
\end{aligned}
$$

Using (4.16) in (4.14) proves the inf-sup property (4.12).

Theorem 4.1. Under the assumptions of Lemma 4.2 there exists a constant $C_{R}>0$, independent of $h$, such that for $u \in W^{2,1}\left(\Omega ; \mathcal{T}_{h}\right)$ it holds

$$
\|R(u)\|_{L^{1}\left(\Omega ; \mathbb{R}^{d \times d}\right)} \leq C_{R} \sum_{E \in \mathcal{E}_{h}(\Omega)} \int_{E}\left|\left[\nabla u \otimes \mathbf{n}_{E}\right]_{E}\right| d s .
$$

Proof. We have

$$
\begin{aligned}
\|R(u)\|_{L^{1}\left(\Omega ; \mathbb{R}^{d \times d}\right)} & =\sup _{\underline{\underline{\varphi}} \in L^{\infty}\left(\Omega ; \mathbb{R}^{d \times d}\right)}\left\{\left(\|\underline{\underline{\varphi}}\|_{L^{\infty}\left(\Omega ; \mathbb{R}^{d \times d}\right)}\right)^{-1} \int_{\Omega} R(u): \underline{\underline{\varphi}} d x\right\} \\
& \geq \sup _{\underline{\underline{\varphi}} \in \underline{\underline{M}}_{h}}\left\{\left(\|\underline{\underline{\varphi}}\|_{L^{\infty}\left(\Omega ; \mathbb{R}^{d \times d}\right)}\right)^{-1} \int_{\Omega} R(u): \underline{\underline{\varphi}} d x\right\} .
\end{aligned}
$$

The inf-sup property (4.12) implies

$$
\|R(u)\|_{L^{1}\left(\Omega ; \mathbb{R}^{d \times d}\right)} \leq C^{-1} \sup _{\underline{\underline{\varphi}} \in \underline{\underline{\mathbf{M}}}_{h}}\left\{\left(\|\underline{\underline{\varphi}}\|_{L^{\infty}\left(\Omega ; \mathbb{R}^{d \times d}\right)}\right)^{-1} \int_{\Omega} R(u): \underline{\underline{\varphi}} d x\right\} .
$$

Now, observing (4.7), we obtain

$$
\begin{aligned}
\int_{\Omega} R(u): \underline{\underline{\varphi}} d x & \leq \sum_{E \in \mathcal{E}_{h}(\Omega)} \int_{E}\left|\left[\nabla u \otimes \mathbf{n}_{E}\right]_{E}\right|\left|\{\underline{\underline{\varphi}}\}_{E}\right| d s \\
& \leq \sum_{E \in \mathcal{E}_{h}(\Omega)} \sup _{x \in E}\left|\{\underline{\underline{\varphi}}(x)\}_{E}\right| \int_{E}\left|\left[\nabla u \otimes \mathbf{n}_{E}\right]_{E}\right| d s \\
& \leq\|\underline{\underline{\varphi}}\|_{L^{\infty}\left(\Omega ; \mathbb{R}^{d \times d}\right)} \sum_{E \in \mathcal{E}_{h}(\Omega)} \int_{E}\left|\left[\nabla u \otimes \mathbf{n}_{E}\right]_{E}\right| d s .
\end{aligned}
$$

Using (4.20) in (4.19) gives (4.17). 


\section{Broken Poincaré-Friedrichs type inequalities}

As a preliminary result we show that for $u \in W^{2,1}\left(\Omega ; \mathcal{T}_{h}\right)$ the second order total variation of $u$ is bounded by the broken $W^{2,1}\left(\Omega ; \mathcal{T}_{h}\right)$ seminorm.

Lemma 5.1. For $u \in W^{2,1}\left(\Omega ; \mathcal{T}_{h}\right)$ it holds

$$
\left|D^{2} u\right|(\Omega) \leq|u|_{W^{2,1}\left(\Omega ; \mathcal{T}_{h}\right)} .
$$

Proof. In view of (2.11), for $\underline{\underline{\varphi}} \in C_{0}^{2}\left(\Omega ; \mathbb{R}^{d \times d}\right)$ with $|\underline{\underline{\varphi}}(x)| \leq 1, x \in \Omega$, we have

$$
-\int_{\Omega} \nabla u \cdot \nabla \cdot \underline{\underline{\varphi}} d x=-\sum_{K \in \mathcal{T}_{h}} \int_{K} \nabla u \cdot \nabla \cdot \underline{\underline{\varphi}} d x .
$$

An element-wise application of Green's formula yields

$$
\begin{aligned}
-\int_{K} \nabla u \cdot \nabla \cdot \underline{\underline{\varphi}} d x & =-\int_{K} \sum_{i, j=1}^{d} \frac{\partial u}{\partial x_{i}} \frac{\partial \varphi_{i j}}{\partial x_{j}} d x \\
& =\int_{K} \sum_{i, j=1}^{d} \frac{\partial^{2} u}{\partial x_{i} \partial x_{j}} \varphi_{i j} d x-\int_{\partial K} \sum_{i, j=1}^{d} \frac{\partial u}{\partial x_{i}} \varphi_{i j} \mathbf{n}_{\partial K, j} d s \\
& =\int_{K} \nabla^{2} u: \underline{\underline{\varphi}} d x-\int_{\partial K} \nabla u \otimes \mathbf{n}_{\partial K}: \underline{\underline{\varphi}} d s,
\end{aligned}
$$

and hence

$$
-\sum_{K \in \mathcal{T}_{h}} \int_{K} \nabla u \cdot \nabla \cdot \underline{\underline{\varphi}} d x=\sum_{K \in \mathcal{T}_{h}} \int_{K} \nabla^{2} u: \underline{\underline{\varphi}} d x-\sum_{E \in \mathcal{E}_{h}} \int_{E}\left[\nabla u \otimes \mathbf{n}_{E}\right]_{E}: \underline{\underline{\varphi}} d s
$$

from which we deduce (5.1).

The previous result implies the following compactness property for the broken Sobolev space $W^{2,1}\left(\Omega ; \mathcal{T}_{h}\right)$.

Theorem 5.1. Let $\left\{u_{h_{n}}\right\}_{\mathbb{N}}$ be a bounded sequence of functions $u_{h_{n}} \in W^{2,1}\left(\Omega ; \mathcal{T}_{h}\right)$, $n \in \mathbb{N}$, where $\left\{h_{n}\right\}_{\mathbb{N}}$ is a sequence of mesh widths $h_{n}>0$ with $h_{n} \rightarrow 0$ as $n \rightarrow \infty$. Then there exist a subsequence $\mathbb{N}^{\prime} \subset \mathbb{N}$ and a function $u \in B V^{2}(\Omega)$ such that

$$
u_{h_{n}} \rightarrow^{*} u\left(\mathbb{N}^{\prime} \ni n \rightarrow \infty\right) \text { in } B V^{2}(\Omega) .
$$

Proof. In view of Lemma 5.1 the sequence $\left\{u_{h_{n}}\right\}_{\mathbb{N}}$ is bounded in $B V^{2}(\Omega)$ and the result follows from Theorem 2.2.

We are now able to derive broken Poincaré-Friedrichs type inequalities in the broken Sobolev space $W^{2,1}\left(\Omega ; \mathcal{T}_{h}\right)$. We set

$$
\begin{aligned}
& V_{h}:=\left\{v \in W^{2,1}\left(\Omega ; \mathcal{T}_{h}\right)|v|_{\Gamma}=0\right\} \\
& W_{m}^{2,1}\left(\Omega ; \mathcal{T}_{h}\right):=\left\{v \in W^{2,1}\left(\Omega ; \mathcal{T}_{h}\right) \mid \int_{\Omega} \frac{\partial v}{\partial x_{i}} d x=0,1 \leq i \leq d\right\} .
\end{aligned}
$$


Theorem 5.2. With the generic constants $C_{i}>0,1 \leq i \leq 2$, from Lemma 3.2 it holds

$$
\begin{array}{ll}
|u|_{W^{1,1}(\Omega)} \leq C_{1}|u|_{W^{2,1}\left(\Omega ; \mathcal{T}_{h}\right)}, \quad & u \in V_{h}, \\
|u|_{W^{1,1}(\Omega)} \leq C_{2}|u|_{W^{2,1}\left(\Omega ; \mathcal{T}_{h}\right)}, & u \in W_{m}^{2,1}\left(\Omega ; \mathcal{T}_{h}\right) .
\end{array}
$$

Proof. The assertions are a direct consequence of the estimates (3.2) and (5.1).

\section{An application to a minimization problem in $B V_{0}^{2}(\Omega)$}

For texture analysis and management in image processing the denoised part $u$ of an image can be obtained as the solution of the minimization problem

$$
F(u)=\inf _{v \in B V_{0}^{2}(\Omega)} F(v),
$$

where the objective functional is given by

$$
F(v):=\frac{1}{2}\left\|v-u_{d}\right\|_{L^{2}(\Omega)}^{2}+\lambda\left|D^{2} v\right|(\Omega) .
$$

Here, $\Omega$ is assumed to be a bounded Lipschitz domain in $\mathbb{R}^{2}$ with boundary $\Gamma=\partial \Omega$, $u_{d} \in L^{2}(\Omega)$ represents the noisy image and $\lambda>0$ is a fidelity parameter. We refer to $[4,5,11]$ for details (cf. also $[15,16]$ for texture analysis based on first order total variation models).

The existence of a solution $u \in B V_{0}^{2}(\Omega)$ can be shown by a minimizing sequence argument (cf., e.g., [4, Theorem 3.4]).

Given a geometrically conforming, simplicial triangulation $\mathcal{T}_{h}$ of $\Omega$ satisfying (4.1), the $\mathrm{C}^{0} \mathrm{DG}$ approximation of (6.1) amounts to the computation of $u \in V_{h}:=\{v \in$ $\left.W^{2,1}\left(\Omega ; \mathcal{T}_{h}\right)|v|_{\Gamma}=0\right\}$ such that

$$
F_{h}(u)=\inf _{v \in V_{h}} F_{h}(v)
$$

with $F_{h}$ referring to the discrete objective functional

$$
\begin{aligned}
F_{h}(v):= & \frac{1}{2}\left\|v-u_{d}\right\|_{L^{2}(\Omega)}^{2}+\lambda \sum_{K \in \mathcal{T}_{h}} \int_{K}\left|\nabla_{h, D G}^{2}\right| d x \\
& +\alpha \sum_{E \in \mathcal{E}_{h}(\Omega)} \int_{E}\left|\left[\nabla v \otimes \mathbf{n}_{E}\right]_{E}\right| d s,
\end{aligned}
$$

where $\nabla_{h, D G}^{2} v$ stands for the DG approximation of the Hessian as given by (4.8) and $\alpha>0$ is a penalty parameter.

Since the broken Sobolev space $V_{h}$ is finite dimensional, for sufficiently large penalty parameter $\alpha>0$ the existence of a solution $u_{h} \in V_{h}$ follows by a standard minimizing sequence argument.

Let $\left\{h_{n}\right\}_{\mathbb{N}}$ be a null sequence of mesh widths $h_{n}>0, n \in \mathbb{N}$. In order to prove that any accumulation point of a sequence $\left\{u_{h_{n}}\right\}_{\mathbb{N}}$ of solutions $u_{h_{n}} \in V_{h_{n}}, n \in \mathbb{N}$, of (6.3) is a solution of (6.1), we prove the following approximation result. 
Lemma 6.1. Let $\left\{h_{n}\right\}_{\mathbb{N}}$ be a null sequence of mesh widths $h_{n}>0, n \in \mathbb{N}$. For any $v \in B V_{0}^{2}(\Omega)$ there exists a sequence $\left\{v_{h_{n}}\right\}_{\mathbb{N}}$ of functions $v_{h_{n}} \in V_{h_{n}} \cap W^{2,1}(\Omega), n \in \mathbb{N}$, such that $\left\|v_{h_{n}}\right\|_{W^{2,1}\left(\Omega ; \mathcal{T}_{h_{n}}\right)} \rightarrow\|v\|_{B V^{2}(\Omega)}$ as $n \rightarrow \infty$.

Proof. In view of Lemma 2.3, without loss of generality we may assume that $u \in C_{0}^{\infty}(\Omega)$. For such a smooth function, the result follows from standard polynomial approximation theory [9].

Theorem 6.1. Let $\left\{h_{n}\right\}_{\mathbb{N}}$ be a null sequence of mesh widths $h_{n}>0, n \in \mathbb{N}$, and let $\left\{u_{h_{n}}\right\}_{\mathbb{N}}$ be a sequence of solutions $u_{h_{n}} \in V_{h}, n \in \mathbb{N}$, of (6.3). For sufficiently large penalty parameter $\alpha$ there exist a subsequence $\mathbb{N}^{\prime} \subset \mathbb{N}$ and a function $u \in B V_{0}^{2}(\Omega)$ such that

$$
u_{h_{n}} \rightarrow^{*} u\left(\mathbb{N}^{\prime} \ni n \rightarrow \infty\right) \text { in } B V^{2}(\Omega),
$$

where $u$ is a solution of (6.1).

Proof. The boundedness of $\left\{F_{h_{n}}\left(u_{h_{n}}\right)\right\}_{\mathbb{N}}$ yields the boundedness of

$$
\left\{\sum_{E \in \mathcal{E}_{h_{n}}(\Omega)} \int_{E}\left|\left[\nabla u_{h_{n}} \otimes \mathbf{n}_{E}\right]_{E}\right| d s\right\}_{\mathbb{N}},
$$

which in view of Theorem 4.1 implies the boundedness of $\left\{R\left(u_{h_{n}}\right)\right\}_{\mathbb{N}}$ in $L^{1}\left(\Omega ; \mathbb{R}^{2 \times 2}\right)$. Moreover, taking (4.17) into account, for $\alpha \geq \lambda C_{R}$ we have

$$
\begin{aligned}
F_{h_{n}}\left(u_{h_{n}}\right) & \geq \lambda \sum_{K \in \mathcal{T}_{h_{n}}} \int_{K}\left|\nabla^{2} u_{h_{n}}\right| d x+\left(\alpha-\lambda C_{R}\right) \sum_{E \in \mathcal{E}_{h_{n}}(\Omega)} \int_{E}\left|\left[\nabla u_{h_{n}} \otimes \mathbf{n}_{E}\right]_{E}\right| d s \\
& \geq \lambda \sum_{K \in \mathcal{T}_{h_{n}}} \int_{K}\left|\nabla^{2} u_{h_{n}}\right| d x
\end{aligned}
$$

from which we deduce the boundedness of $\left\{\sum_{K \in \mathcal{T}_{h_{n}}} \int_{K}\left|\nabla^{2} u_{h_{n}}\right| d x\right\}_{\mathbb{N}}$. Consequently, we obtain the boundedness of $\left\{\left|u_{h_{n}}\right|_{W^{2,1}\left(\Omega ; \mathcal{T}_{h_{n}}\right)}\right\}_{\mathbb{N}}$. Due to the broken Poincaré-Friedrichs inequality (5.4a) from Theorem 5.2, the sequence $\left\{\left\|u_{h_{n}}\right\|_{W^{2,1}\left(\Omega ; \mathcal{T}_{h_{n}}\right)}\right\}_{\mathbb{N}}$ is bounded. Theorem 5.1 implies the existence of a subsequence $\mathbb{N}^{\prime} \subset \mathbb{N}$ and a function $u \in B V^{2}(\Omega)$ such that

$$
u_{h_{n}} \rightarrow^{*} u\left(\mathbb{N}^{\prime} \ni n \rightarrow \infty\right) \text { in } B V^{2}(\Omega)
$$

In particular, we have

$$
D^{2} u_{h_{n}} \rightarrow^{*} D^{2} u\left(\mathbb{N}^{\prime} \ni n \rightarrow \infty\right) \text { in } \mathcal{M}\left(\Omega ; \mathbb{R}^{2 \times 2}\right) .
$$

Due to the boundedness of $\left\{\sum_{K \in \mathcal{T}_{h_{n}}} \int_{K}\left|\nabla^{2} u_{h_{n}}\right| d x\right\}_{\mathbb{N}}$ and $\left\{R\left(u_{h_{n}}\right)\right\}_{\mathbb{N}}$ in $L^{1}\left(\Omega ; \mathbb{R}^{2 \times 2}\right)$ there exist a subsequence $\mathbb{N}^{\prime \prime} \subset \mathbb{N}^{\prime}$ and $\underline{\underline{\mathbf{g}}}_{i} \in \mathcal{M}\left(\Omega ; \mathbb{R}^{2 \times 2}\right), 1 \leq i \leq 2$, such that

$$
\nabla_{h}^{2} u_{h_{n}} \rightarrow^{*} \underline{\underline{g}}_{1}, \quad R\left(u_{h_{n}}\right) \rightarrow^{*} \underline{\underline{g}}_{2}\left(\mathbb{N}^{\prime \prime} \ni n \rightarrow \infty\right) \quad \text { in } \mathcal{M}\left(\Omega ; \mathbb{R}^{2 \times 2}\right) \text {. }
$$


We show that the jumps generate $\underline{\underline{g}}_{2}$ in the sense that for $\underline{\underline{\varphi}} \in C_{0}^{\infty}\left(\Omega ; \mathbb{R}^{2 \times 2}\right)$ it holds

$$
-\sum_{E \in \mathcal{E}_{h_{n}}(\Omega)} \int_{E}\left[\nabla u_{h_{n}} \otimes \mathbf{n}_{E}\right]_{E}: \underline{\underline{\varphi}} d s \rightarrow \int_{\Omega} \underline{\underline{\mathbf{g}}}_{2}: \underline{\underline{\varphi}} d x \quad\left(\mathbb{N}^{\prime \prime} \ni n \rightarrow \infty\right) .
$$

Let $\left\{\underline{\underline{\varphi}}_{h_{n}}\right\}_{\mathbb{N}^{\prime \prime}}$ be a sequence of matrices $\underline{\underline{\varphi}}_{h_{n}} \in \underline{\underline{\mathbf{M}}}_{h_{n}}, n \in \mathbb{N}^{\prime \prime}$, such that

$$
\left\|\underline{\underline{\varphi}}-\underline{\underline{\varphi}}_{h_{n}}\right\|_{L^{\infty}\left(\Omega ; \mathbb{R}^{2 \times 2}\right)} \rightarrow 0 \quad\left(\mathbb{N}^{\prime \prime} \ni n \rightarrow \infty\right) .
$$

In view of (4.7) it follows that

$$
\begin{aligned}
& -\sum_{E \in \mathcal{E}_{h_{n}}(\Omega)} \int_{E}\left[\nabla u_{h_{n}} \otimes \mathbf{n}_{E}\right]_{E}: \underline{\underline{\varphi}} d s \\
= & \left.-\sum_{E \in \mathcal{E}_{h_{n}}(\Omega)} \int_{E}\left[\nabla u_{h_{n}} \otimes \mathbf{n}_{E}\right]_{E}: \underline{\underline{\varphi}}-\underline{\underline{\varphi}}_{h_{n}}\right) d s-\sum_{E \in \mathcal{E}_{h_{n}}(\Omega)} \int_{E}\left[\nabla u_{h_{n}} \otimes \mathbf{n}_{E}\right]_{E}: \underline{\underline{\varphi}}_{h_{n}} d s \\
= & \left.-\sum_{E \in \mathcal{E}_{h_{n}}(\Omega)} \int_{E}\left[\nabla u_{h_{n}} \otimes \mathbf{n}_{E}\right]_{E}: \underline{\underline{\varphi}}-\underline{\underline{\varphi}}_{h_{n}}\right) d s+\int_{\Omega} R\left(u_{h_{n}}\right): \underline{\underline{\varphi}}_{h_{n}} d x \\
= & \left.-\sum_{E \in \mathcal{E}_{h_{n}}(\Omega)} \int_{E}\left[\nabla u_{h_{n}} \otimes \mathbf{n}_{E}\right]_{E}: \underline{\underline{\varphi}}-\underline{\underline{\varphi}}_{h_{n}}\right) d s+\int_{\Omega} R\left(u_{h_{n}}\right):\left(\underline{\underline{\varphi}}_{h_{n}}-\underline{\underline{\varphi}}\right) d x \\
& +\int_{\Omega} R\left(u_{h_{n}}\right): \underline{\underline{\varphi}} d x .
\end{aligned}
$$

The first and second term on the right-hand side of (6.10) tend to zero as $\mathbb{N}^{\prime \prime} \ni n \rightarrow \infty$. In view of (6.8) and (6.9) it follows that $D^{2} u=\underline{\underline{\mathbf{g}}}_{1}+\underline{\underline{\mathbf{g}}}_{2}$ and hence, we have

$$
\nabla_{h_{n}, D G}^{2} u_{h_{n}} \rightarrow^{*} D^{2} u\left(\mathbb{N}^{\prime \prime} \ni n \rightarrow \infty\right) \text { in } \mathcal{M}\left(\Omega ; \mathbb{R}^{2 \times 2}\right) .
$$

In view of Lemma 3.1 we have $\left.\left.u_{h_{n}}\right|_{\Gamma} \rightarrow u\right|_{\Gamma}\left(\mathbb{N}^{\prime \prime} \ni n \rightarrow \infty\right)$ in $L^{1}(\Gamma)$ and hence, it follows that $u \in B V_{0}^{2}(\Omega)$.

It remains to be shown that $u \in B V_{0}^{2}(\Omega)$ is a solution of (6.1). Taking (6.11) into account, the lower semicontinuity result Theorem 2.3 infers

$$
\left|D^{2} u\right|(\Omega) \leq \lim \inf _{\mathbb{N}^{\prime \prime} \ni n \rightarrow \infty} \sum_{K \in \mathcal{T}_{h_{n}}} \int_{K}\left|\nabla_{h_{n}, D G}^{2} u_{h_{n}}\right| d x .
$$

Moreover, since for $d=2$ the space $B V^{2}(\Omega)$ is compactly embedded in $L^{2}(\Omega)$ (Theorem 2.3), we have

$$
u_{h_{n}} \rightarrow u\left(\mathbb{N}^{\prime \prime} \ni n \rightarrow \infty\right) \text { in } L^{2}(\Omega),
$$

and hence, it follows that

$$
\left\|u_{h_{n}}-u_{d}\right\|_{L^{2}(\Omega)}^{2} \rightarrow\left\|u-u_{d}\right\|_{L^{2}(\Omega)}^{2} \quad \text { as } \quad \mathbb{N}^{\prime \prime} \ni n \rightarrow \infty .
$$


As a consequence of (6.12) and (6.14) we have

$$
F(u) \leq \lim \inf _{\mathbb{N}^{\prime \prime} \ni n \rightarrow \infty}\left(\frac{1}{2}\left\|u_{h_{n}}-u_{d}\right\|_{L^{2}(\Omega)}^{2}+\lambda \sum_{K \in \mathcal{T}_{h_{n}}} \int_{K}\left|\nabla_{h_{n}, D G}^{2} u_{h_{n}}\right| d x\right) .
$$

Now, for a fixed, but arbitrarily chosen $v \in B V_{0}^{2}(\Omega)$, in view of Lemma 6.1 there exists a sequence $\left\{v_{h_{n}}\right\}_{\mathbb{N}}$ of functions $v_{h_{n}} \in V_{h_{n}} \cap W^{2,1}(\Omega), n \in \mathbb{N}$, such that $\left\|v_{h_{n}}\right\|_{W^{2,1}\left(\Omega ; \mathcal{T}_{h_{n}}\right)} \rightarrow$ $\|v\|_{B V^{2}(\Omega)}$ as $n \rightarrow \infty$. Again, since $B V^{2}(\Omega)$ is compactly embedded in $L^{2}(\Omega)$, we have that the sequence $\left\{v_{h_{n}}\right\}_{\mathbb{N}}$ converges strongly to $v$ in $L^{2}(\Omega)$ as $n \rightarrow \infty$, whence

$$
F_{h_{n}}\left(v_{h_{n}}\right) \rightarrow F(v) \text { as } n \rightarrow \infty .
$$

Since the penalty term in (6.4) vanishes for $v_{h_{n}}$, from (6.15) and (6.16) we deduce

$$
\begin{aligned}
F(u) & \leq \lim \inf _{\mathbb{N}^{\prime \prime} \ni n \rightarrow \infty}\left(\frac{1}{2}\left\|u_{h_{n}}-u_{d}\right\|_{L^{2}(\Omega)}^{2}+\lambda \sum_{K \in \mathcal{T}_{h_{n}}} \int_{K}\left|\nabla_{h_{n}, D G}^{2} u_{h_{n}}\right| d x\right) \\
& \leq \lim \sup _{\mathbb{N}^{\prime \prime} \ni n \rightarrow \infty}\left(\frac{1}{2}\left\|v_{h_{n}}-u_{d}\right\|_{L^{2}(\Omega)}^{2}+\lambda \sum_{K \in \mathcal{T}_{h_{n}}} \int_{K}\left|\nabla_{h_{n}, D G}^{2} v_{h_{n}}\right| d x\right) \\
& =\lim \sup _{\mathbb{N}^{\prime \prime} \ni n \rightarrow \infty} F_{h_{n}}\left(v_{h_{n}}\right)=F(v),
\end{aligned}
$$

which shows that $u \in B V_{0}^{2}(\Omega)$ is a solution of (6.1).

\section{Acknowledgments}

The work was supported by the NSF grant DMS-1520886.

\section{References}

[1] L. Ambrosio, N. Fusco, and D. PAllara, Functions of Bounded Variation and Free Discontinuity Problems, Oxford University Press, 2000.

[2] H. Aтtouch, G. Buttazzo, And G. Michaille, Variational Analysis in Sobolev and BV Spaces, Vol. 6 of the MPS/SIAM Series on Optimization, SIAM, 2006.

[3] M. Bergounioux, On Poincaré-Wirtinger inequalities in BV spaces, Control. Cybern., 4 (2011) 921-929.

[4] M. BERgOUnIOUX, Second-order variational models for image texture analysis, Adv. Imaging Electron Phys., 181 (2014) 35-124.

[5] M. BERgounioux AND L. PIFFET, A full second order model for multiscale texture analysis, Comput. Optim. Appl., 54 (2013) 215-237.

[6] S.C. BRENNER, Poincaré-Friedrichs inequalities for piecewise H1 functions, SIAM J. Numer. Anal., 41 (2003) 306-324.

[7] S.C. BRENNER, Korns inequalities for piecewise $H 1$ vector fields, Math. Comput., 73 (2004) 1067-1087. 
[8] A. Buffa AND C. ORTNER, Compact embeddings of broken Sobolev spaces and applications, IMA J. Numer. Anal., 29 (2009) 827-855.

[9] P. G. Ciarlet, The Finite Element Method for Elliptic Problems, SIAM, 2002.

[10] F. DemEnGEL, Fonctions à hessien borné, Annales de l'Institut Fourier, 34 (1984) 155-190.

[11] W. HintERBERGER AND O. SCHERZER, Variational methods on the space of functions of bounded Hessian for convexification and denoising, Computing, 76 (2006) 109-133.

[12] A. LASIS AND E. SÜLI, Poincaré-type inequalities for broken sobolev spaces, Technical Report 03/10, Oxford University Computing Laboratory, 2003.

[13] C. ORTNER AND E. SÜLI, Discontinuous Galerkin finite element approximation of nonlinear second-order elliptic and hyperbolic systems, SIAM J. Numer. Anal., 45 (2007) 1370-1397.

[14] L. TARTAR, Introduction to Sobolev Spaces and Interpolation Theory, Springer, 2007.

[15] L. A. VeSE AND S. OSHER, Modeling textures with total variation minimization and oscillating patterns in image processing, J. of Sci. Comput., 19 (2003) 553-572.

[16] L. A. VeSE AND S. OSHER, Image denoising and decomposition with total variation minimization and oscillatory functions, J. Math. Imaging Vision, 20 (2004) 7-18. 